\title{
NOTICIAS
}

PRIMER CONGRESO INTERNACIONAL SOBRE EL CONDUCTISMO Y LAS CIENCIAS DE LA CONDUCTA

Guadalajara, México, octubre 5-9 de 1992.

'Toda solicitud de información debe enviarse al profesor Peter Harzem, Department of Psychology, Auburn University, Auburn Alabama $36849-4412$, EEUL (lengua inglesa); o al profesor Emilio Ribes Iñesta, Centro de Estudios e Investigaciones en Psicología, Universidad de Guadalajara, 12 de diciembre 204, Apartado Postal 5-374, Col Chapalita, Guadalajara, Jalisco, 45030, Méxíco.

HISTORIA DE LAS CIENCIAS FÍSICO-MATEMÁTICAS Y ENSEÑANZA DE IAS CIENCIAS

Universidad Complutense, Madrid.9-11 de septiembre 1992.

El Grupo Interdivisional de Historia de la Física de la Sociedad Furopea de Física en colaboración con el Departamento de Didáctica de las Ciencias Experimentales de la Universidad Complutense de Madrid está organizando un congreso internacional dirigido a estudiantes, profesores e investigadores interesados en la historia de la ciencia, la enseñanza de las Ciencias físico-matemáticas y la formación de los profesores.

Las sesiones inclutrán:

- Simulación y reproducción de experimentos científicos.

- Los textos científicos como recurso didáctico.

- Archivos, instrumentos y museos de ciencias aplicados a la enseñanza de las ciencias.

- Dificultades conceptuales y de procedimiento en la enseñanza/aprendizaje de las ciencias físico-matemáticas.
- La historia de las ciencias físico-matemáticas en la formación inicial y permanente del profesorado.

Para recibir la segunda circular deI Congreso, se ruega que envíen los datos de la ficha adjunta a la siguiente direccion:

Dr. D. Antonio Moreno González

Departamento de Didáctica de las Ciencias Experimentales. Escuela Universitaria de Formación de Profesores «María Díaz Jiménez»

Avda. Filipinas, 3.8003

Madrid (España)

Preinscripción: Antes del 31 de enero de 1992.

Propuestas de participación (comunicación, talleres, paneles): Antes del 30 de marzo de: 1992.

Inscripción definitiva: Antes del $30 \mathrm{dc}$ junio de 1992.

Cuota de inscripción: 10.000 pesetas.

CONGRESO LATINOAMERICANO DE HISTORIA DE IAA CIENCIA Y DE LA TECNOLOGÍA

\section{Ciudad de México}

12-16 de enero de 1992.

La reunion se efectuará bajo el patrocinio de la Sociedad Latinoamericana de Historia de las Ciencias y la Tecnología (SLHCT) y de la Sociedad Mexicana de Historia de la Ciencia y de la Tecnología (SMHCT). El tema general del Congreso serấ: "Anérica en la formación de un mundo nuevo: 500 años de intercambios cientificos». En esta oportunidad sc realizará también un Simposio Internacional sobre el «Bicentenario del Real Seminario de Minería de la Ciudad de México».

Cuota de inscripción: 150 dolares.

Interesadus dirigir la correspondencia a: Comité Organizador deI ILI CLAHCI

Apartado Postal 2 I-873

04000 México, D.F. México

\section{LAS DIDÁCTICAS ESPECÍFICAS EN LA FORMACION DEL PROFE- SORADO}

Conferencia internacional para la definición de un ámbito curricular.

Organizada por el Departamento de Didacticas específicas y de Didáctica general de la Universidad de Santiago de Compostela tiene como finalidades:

- Determinar la contribución de las didácticas específicas a los procesos formativos de todos los profesores.

- Facilitar la articulación de las didacticas especificas con el resto de los ámbitos curriculares de la formación de profesores, y especialmente con aquellos campos de contenido incluidos en el campo de la didáctica general.

-Posibilitar una mayor interacción entre la teoría y la práctica.

Indagar la identidad epistemológica de las didácticas específicas.

--Contribuir a un mejor conocimiento de las distintas realidades, con especial atención a la europea, en lo respecta a este ámbito curricular.

Estos propósitos servirán como núcleos temáticos en torno a los que se organizarán las diversas actividades.

Puede dirigirse la correspondencia a:

Lourdes Montero Mesa

Facultade de Filosofia e Ciencias da Educacion

Tel. 563 I 00 ext. 2043

Universidade da Santiago de Compostela

Campus Sur.

Santiago de Compostela (España)

o bien a:

José Manuel Vez Jeremías

Escola Universitaria de Maxisterio

Tel. 583458

Universidade de Santiago de Compostela Avda. Xoán XXIII s/n

15704 Santiago de Compostela (España). 


\section{SEGUNIDA CONFERENCIA INTER- NACIONAL SOBRE HISTORIA $Y$ FIIOSOFIA DE LA CIENCIA EN LA ENSENANZA DE LAS CIENCIAS}

II a 15 de mayo de 1992. Universidad de Queen, Kingston, Ontario, Canadá.

La primera relación de participantes es apabullante si se tiene en cuenta que podrian reunirse en los cinco días de la conferencia profesionales tan destacados como Derek Hodson, Joseph Novak, Joan Solomon, John Olson, Glen Aikenhead, George Posner, Ken T'obin, Pinchas Tamir y Daniel Gil entre los casi cuarenta ponentes, algunos de ellos pendientes de confirmación.

El importe de la inscripción es de 250 dólares canadienses, antes del 15 de febrero de 1992; y de 300 dolares, con posterioridad a dicha fecha.

Para cualquier información dirigirse a:
Dr. Skip Hills

Faculty of Education, Queen's Lniversity Kingston, Ontario, Canada

K7L 3N6

Tel. 1-613-545-6260

FAX 1-613-545-6584

ENSEÑANZA DE LA FÍSICA MODERNA-FÍSICA ESTADÍSTICA

Del 19 al 26 de julio de 1992 se celebrará en Badajoz, en el marco de la Universidad de Exiremadura, la Conferencia Internacional de la Comisión C-14 de la IUPAP deditada a la enseñanza de la Fisica Mode:na.
El propósito de la conferencia es proporcionar un marco de encuentro, donde expertos en investigación, en las áreas que configuran la HISICA ESTADíSTICA Y LA TERMODINÁMICA, en sentido amplio, junto con profesores $y$ especialistas de varios niveles educativos, intercambien ideas y propongan nuevos materiales, métodos y experiencias.

La presentación de resultados de investigaciones en la ensenanza de estas áreas y Ja elaboración de nucvos matejiales didácticos por parte de comisiones de ámbito internacional son los objetivos básicos de la conferencia.

Los trabajos se llevarán i cabo en grupos previamente conslituidos, por cuyo motivo la participación será limitida. la lengua oficial será el inglés, aunque las sesiones amplias gozarán de traducción simultánea. Habrá exposición de pancies y carteles con los trabajos de los asistentes. 